\title{
Low voltage tunable liquid crystal lens
}

\author{
Fan Fan, Abhishek Kumar Srivastava,* Tao Du, Man Chun Tseng, Vladimir Chigrinov, and Hoi Sing Kwok \\ Center for Display Research, Department of Electrical and Electronic Engineering, The Hong Kong University \\ of Science and Technology, Clear Water Bay, Kowloon, Hong Kong, China \\ *Corresponding author: abhishek_srivastava_lu@yahoo.co.in
}

Received July 25, 2013; revised September 4, 2013; accepted September 5, 2013;

posted September 9, 2013 (Doc. ID 194620); published October 9, 2013

\begin{abstract}
In this Letter, we report a method to prepare a liquid crystal (LC) lens based on a variable pretilt angle of a photoalignment (PA) layer. With precise control of the irradiance, such PA provides good control on the pretilt angle that can be tuned in the range of $1^{\circ}-89^{\circ}$. Therefore, the precise control of the irradiance generates a spatially varying pretilt angle with any desired pretilt angle profile. Thus, the tunable LC lenses have been fabricated and characterized. With low voltage operations, easy fabrication, and relatively high switching speed, such lenses can be applied in many modern optical and photonic devices. (c) 2013 Optical Society of America

OCIS codes: (160.3710) Liquid crystals; (230.3720) Liquid-crystal devices; (220.3630) Lenses.

http://dx.doi.org/10.1364/OL.38.004116
\end{abstract}

Liquid crystal (LC) lenses have been explored extensively in recent years [1-5]. Because of electrically tunable focal lengths, these lenses have many applications, like three-dimensional displays, imaging systems, microscopes, zooming systems, optical tweezers, etc. [4-9]. Compactness in size and easy tunability provides an edge for such lenses to be applied in portable mobile devices, like cell phones, cameras, and pico projectors. However, the power consumption, aperture, and switching time are still serious issues, particularly for portable devices. Several attempts have recently been made to improve the characteristics of such lenses [1-9]. The main idea for the tunable LC lens is to provide the lens-like phase profile, i.e., converging or diverging to the propagating wave. The most common approach is to control the LC director by means of a nonuniform electric field that could be done by the nonhomogeneous cell gap, curved or patterned electrodes, and by some nonuniform alignment. However, all of these approaches deal with complicated fabrication procedure, high power consumption, and poor optical performance [ $\underline{6}-\underline{9}]$.

In a different approach, variation of the anchoring energy or pretilt angle has been proposed to fabricate low power consuming LC lenses. However, providing quantized distribution of nonuniform anchoring energy is a real challenge for the community. On the other hand, spatially varying the pretilt angle can be a good alternative to achieve low power consuming tunable LC lenses. The pretilt angle of the LC alignment is an important parameter to define the characteristic of LC devices. Usually, most of the LC devices are based on either planar or vertical alignment that corresponds to the pretilt angle values $0^{\circ}-10^{\circ}$ or $80^{\circ}-90^{\circ}$. Achieving stable pretilt angles between $10^{\circ}$ and $80^{\circ}$ is still difficult [10]. In recent years, several LC applications, such as bistable display [11], nobias-bend fast switching display [12], LC tunable lenses, [13] etc. appeared, which demand a pretilt angle in the range of $10^{\circ}-80^{\circ}$. Therefore, several methods to generate the broad range of pretilt angle have been proposed that include mixing of planar and vertical alignment material $[\underline{10}, \underline{14}]$, mixing of polyamic acids [15], stacked alignment layer $[\underline{13}, \underline{16}]$, oblique evaporation of $\mathrm{SiO}_{2}$ [17], etc.

Recently, Tseng et al. reported LC lenses based on varying pretilt angles fabricated by the stacked alignment (two or more alignment layers) layer [13]. These lenses provide low voltage operations. However, the stacked alignment layer fabrication is rather complicated and imposes certain limitations for the alignment quality and fabrication procedure. Here, we report a tunable LC lens based on the approach of controlling the pretilt angle profile by means of the irradiance doses of a photoalignment (PA) layer. Such a PA layer provides precise control on the pretilt angle over a wide range of the pretilt angles, i.e., $1^{\circ}-89^{\circ}$, which provides a great potential for applications not only in the tunable LC lens but also for other photonics and display devices.

The PA material CPL024 [from Dai-Nippon Ink and Chemicals (DIC)], which is a cross-linking polymer, provides a broad range of pretilt angles $\left(1^{\circ}-89^{\circ}\right)$ depending on the irradiance doses. The pretilt angle profile for the CPL024 at different irradiance has been studied by making a $10-\mu \mathrm{m}$ thick-antiparallel LC cell with several distinct areas with different irradiance (Fig. 1). The fabrication process includes the following steps. First, the CPL024 powder was dissolved in solvent cyclohexanone with a concentration of $0.5 \% \mathrm{wt} / \mathrm{wt}$. Then, the solution was spin-coated on ITO glass substrates and thereafter exposed by UV light, of the wavelength $328 \mathrm{~nm}$, at a plane inclined obliquely at an angle of $45^{\circ}$. This oblique exposure defines the azimuthal direction $\varphi$ (defined in Fig. 1) of the alignment layer which is critically important to generate such a regular pretilt angle profile; otherwise it will be random. The fabrication process flow is shown in the top insertion of Fig. 1.

Thereafter, the crystal rotating method was used to measure the pretilt angles for different areas with different irradiance; its dependence is shown in Fig. 1. The pretilt angle increases with an increase in irradiance. The inset at the bottom right of Fig. 1 represents the photos for the LC cell with different pretilt angles achieved at different irradiance, which confirms the good alignment quality with different pretilt angles. The slight change in effective birefringence and the cell gap can be attributed to the variation in brightness and color appearance for different exposure times. Moreover, the achieved pretilt angle is pretty stable against temperature and other environmental conditions. Almost no changes have been observed, even after several days. The pretilt was measured 


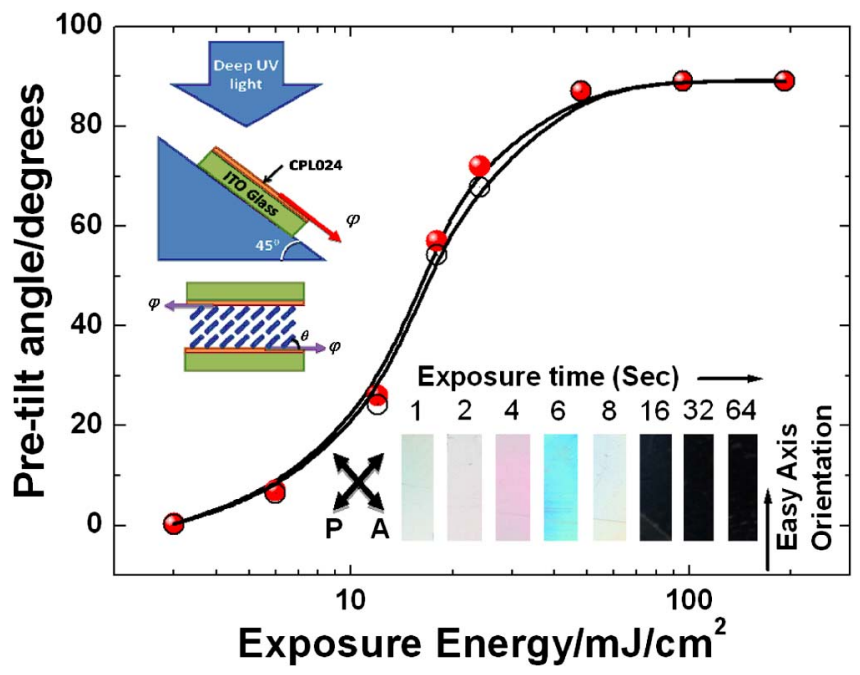

Fig. 1. Pretilt angle dependence on the exposure energy (solid circles). Open legends represent the same experimental results repeated after several hours of photographing and thermal exposure. The top insertion shows the simple fabrication procedure to make the LC cell to analyze different PA parameters, whereas the bottom insertion shows the photograph of the prepared LC cell under the crossed polarizers for distinct areas with different irradiance and pretilt angles.

again after several hours of photographing with the thermal exposure at $100^{\circ} \mathrm{C}$. The deviation in the two experiments is within the experimental error and reveals material stability.

The pretilt angle depends on the irradiance. Therefore, spatially variable UV irradiance patterns can be employed to achieve any desired pattern. The same approach has been used to fabricate the LC tunable lens. The fabrication process to fabricate the LC lens is as follows. First, the CPL024-coated ITO glass substrate was exposed obliquely for $1 \mathrm{~s}$ using the setup showed in Fig. 1 to define the preferred azimuthal direction $\varphi$, which provides the pretilt angle of $\sim 1^{\circ}$. Afterward, one of the two substrates was exposed further, with the same setup, for $32 \mathrm{~s}$ more, which produced the pretilt angle of $\sim 89^{\circ}$. However, the second substrate was exposed normally by the same UV source but through a mask with a circular aperture. The schematics of the experimental setup and cell structure are shown in Figs. 2(a) and 2(b), respectively.

To generate the spatially variable $\overline{\mathrm{UV}}$ irradiance, a mask with circular structure of radius $R$ was placed at a distance $D$ from the substrate. Due to the angular divergence $(\beta)$ of the UV light source (i.e., $\sim 5^{\circ}$ ), the UV light will leak onto the substrate surface from the border of the mask. When $R / D=\tan \beta$, the light intensity exposed on the substrate will be distributed in circular symmetry with the smallest intensity at the center and the largest at the border of the circle. Therefore, after being exposed through this irradiance pattern, the pretilt angle defined on the substrate will be spatially variable with the smallest pretilt angle $\left(\sim 1^{\circ}\right)$ at the center and increasing gradually to the largest pretilt angle $\left(\sim 89^{\circ}\right)$ at the border.

In the next step, the LC cell was assembled with the prepared substrates in an antiparallel azimuthal arrangement. The cell gap was maintained at $15 \mu \mathrm{m}$, and a nematic LC RDP97304 (from DIC Corporation) with (a)

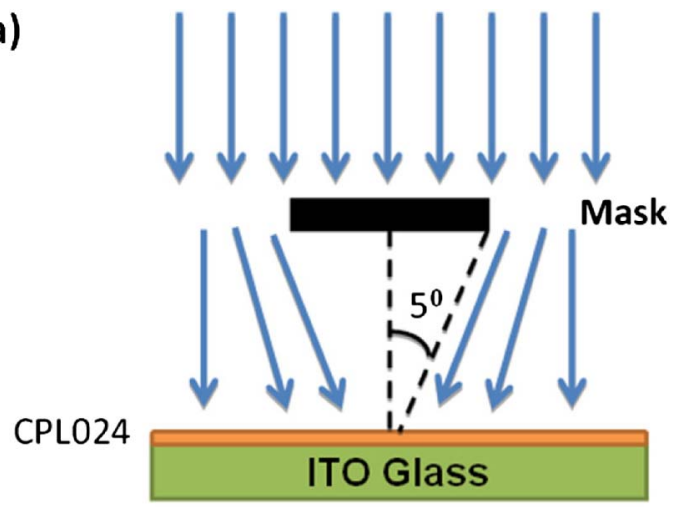

(b)

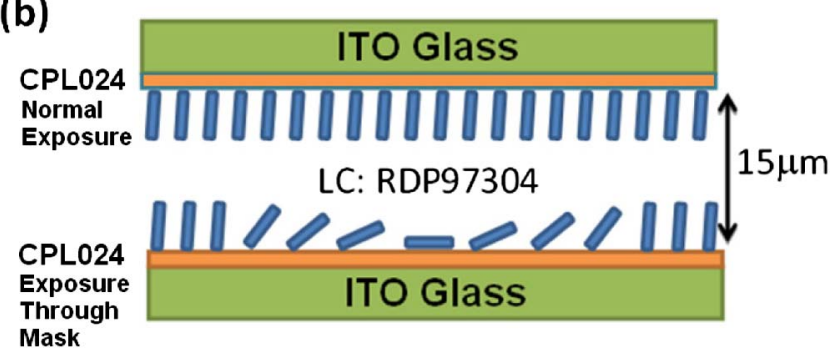

Fig. 2. (a) Exposure setup to achieve spatially variable light intensity on the exposing plane. (b) The fabricated LC lens structure.

$\delta n=0.27$ was filled into the LC cell. The fabricated tunable LC lens structure is shown in Fig. 2. This LC lens is polarization dependent. For the polarization plane parallel to the azimuthal direction of alignment, the LC cell has spatially variable phase value, while in the perpendicular direction it has a uniform phase profile. Nevertheless, this polarization dependence is not the issue and can be resolved by two or multiple stacks $[\underline{18}, \underline{19}]$.

In order to characterize the fabricated tunable LC lens, the phase profile in the direction that is parallel to the azimuthal direction has been measured. Polarization microscopy was used to analyze the phase profile. A red filter with a transmission peak at $650 \mathrm{~nm}$ was used to filter the white light source to narrow band light. Thereafter, the fabricated LC lens was placed between crossed polarizers with the azimuthal direction at $45^{\circ}$ in the plane. After that, an AC signal with frequency $1 \mathrm{kHz}$ was applied to tune the LC lens. The microscopic photographs of the LC lens at different voltage are shown in Fig. 3, wherein the length of the marker is $700 \mu \mathrm{m}$.

The transmittance at different points can be calculated as

$$
T=\sin ^{2}\left(\frac{\pi d \Delta n}{\lambda}\right)
$$

where $d$ is the LC cell gap, $\Delta n$ is the average birefringence, and $\lambda$ is the light wavelength. The transmittance value was extracted from the photos shown in Fig. 3, and Eq. (1) was used to calculate the retardation parameter profile where the retardation parameter is defined as $\delta=\pi d \Delta n / \lambda$. The retardation parameter profiles for the fabricated circular LC lens at different voltages are shown in Fig. 4. All of these retardation parameter 


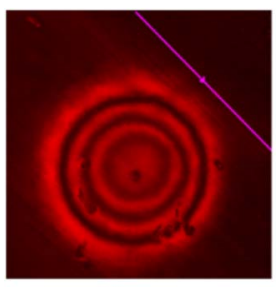

oV

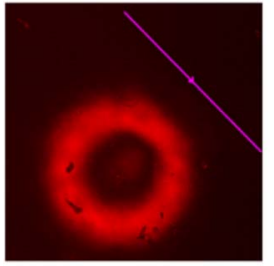

$1.5 \mathrm{~V}$

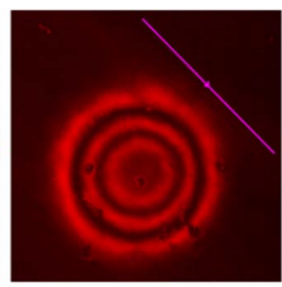

$0.5 \mathrm{~V}$

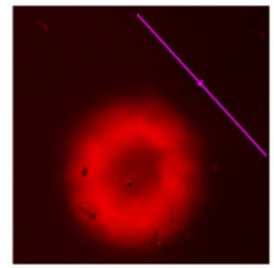

2V

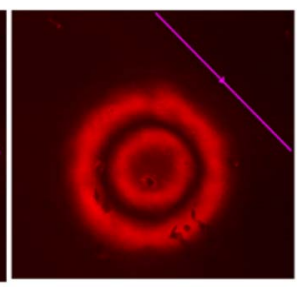

1V

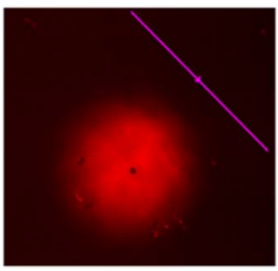

3V
Fig. 3. Optical microphotographs of the circular LC lens at different applied voltages that have been clicked in red light. The marker size is $700 \mu \mathrm{m}$.

profiles show parabolic character that is also the best case for any lens design [18]. The solid lines in Fig. $\underline{4}$ represent the best fit of the parabola approximation to the experimental retardation profile (open legends). It is clear from Fig. $\underline{4}$ that the retardation parameter profiles fit very well with the parabola approximation, which confirms the working concept of the designed LC lens.

The focal length $f$ of the LC lens could be calculated by the equation

$$
f=\frac{r^{2}}{2 d\left(n_{e 2}-n_{e 1}\right)},
$$

where $r$ is the radius of the LC lens, $d$ is the cell gap, and $n_{e 2}-n_{e 1}$ represents the effective refractive index difference between the center and the border of the LC lens. Using Eq. (2), $f$ can be calculated along with

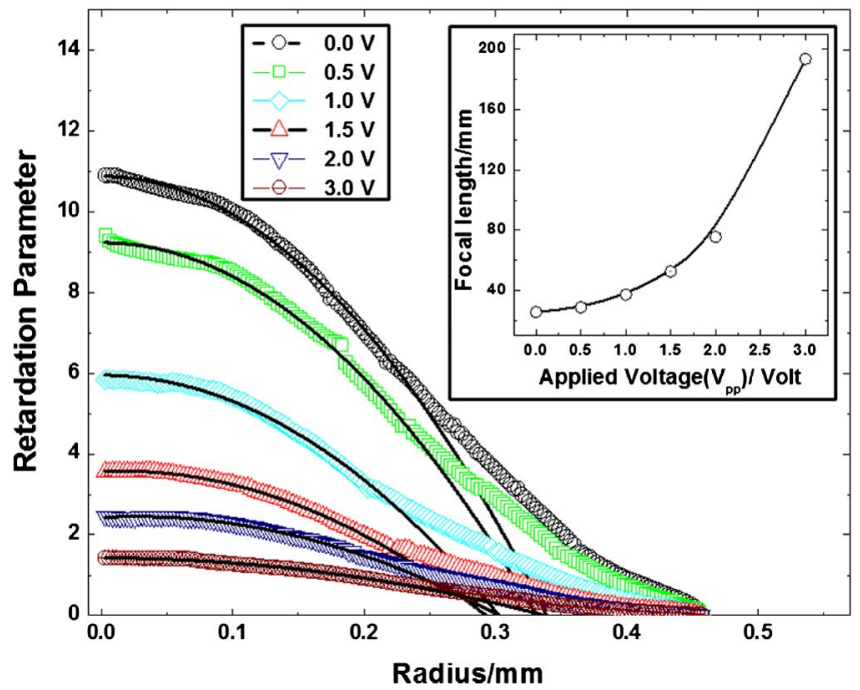

Fig. 4. Retardation parameter profile dependence along the radius of the $\mathrm{LC}$ lens at different voltages. The open legends show the experimental retardation parameter profile, while the solid lines represent the best parabolic fitting. The inset shows the voltage dependence of the focal length of the same lens. the retardation parameter profile shown in Fig. 4. The voltage dependence of $f$ is shown in the inset of Fig. 4 . It can be seen that when no voltage is applied, the focal length is $25 \mathrm{~mm}$, and it increases at higher applied voltages. Moreover, the focal length can be tuned further on applying a higher electric field and could become infinite on applying a sufficiently large voltage. Thus, the focal length of the fabricated lens can be tuned in a broad range by applying a relatively smaller electric field. Only $3 \mathrm{~V}$ is enough to tune $f$ from 2.5 to $20 \mathrm{~cm}$, which can be decreased further by optimizing the LC parameters and cell gap of the lens.

Furthermore, several other pretilt angle profiles and devices could be fabricated by the same methodology. To confirm this, a cylindrical lens was fabricated by using the rectangular mask, and its operation is shown by microphotographs at different applied voltages in Fig. 5 .

In this Letter, we reported an approach to generate the spatially variable pretilt angle profile that is used to design the low voltage tunable LC lens. The PA technique has been employed to generate the lens profile. By changing the irradiance, the pretilt angle can be tuned in a broad range (i.e., $1^{\circ}-89^{\circ}$ ). All lenses, because of such pretilt angle profiles, can be controlled by an extremely low electric field, which is the real advantage of such an approach, and a lower cell gap provides relatively faster electro-optical modulation speed. Moreover, focal length can be tuned in a broad range (i.e., from the predefined $f$, by the fabrication parameters, to $\propto$ ) at a relatively lower

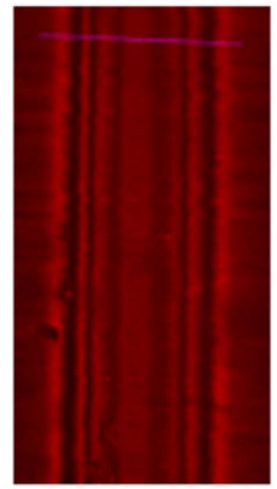

\section{OV}

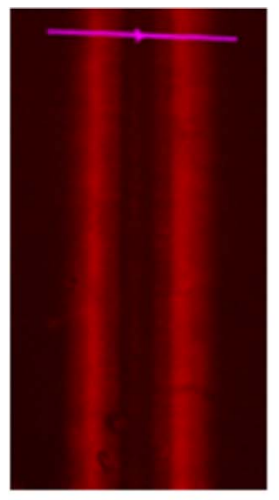

$1.5 \mathrm{~V}$

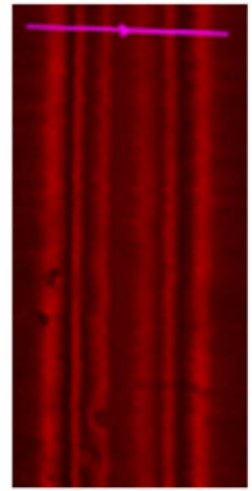

$0.5 \mathrm{~V}$

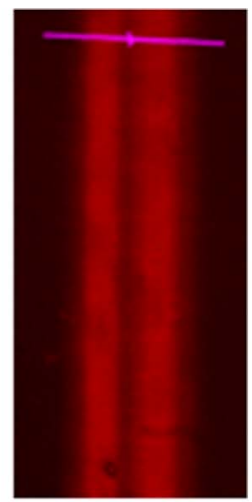

2V

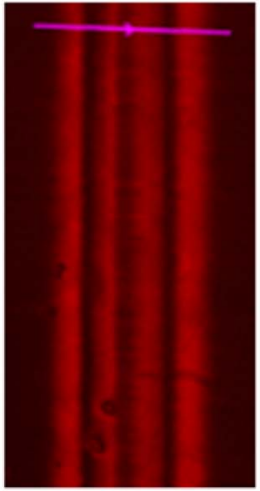

1V

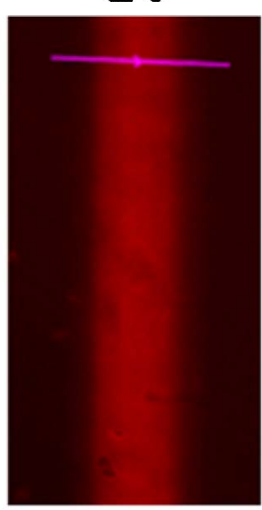

3V
Fig. 5. Optical microphotograph of the cylindrical LC lens at different applied voltages that have been clicked in red light. The marker size is $400 \mu \mathrm{m}$. 
electric field. Optimizing the LC and cell parameters can decrease the operating voltages further. In addition to this, with precise control on parameters of the exposing mask, the distance between the mask and substrate, and angular divergence of the exposing light source, one can achieve any desired lens profile and aperture ratio. Furthermore, given the easy fabrication procedure in association with low operating voltages, such devices could find applications in many modern optical and photonic devices. The proposed approach can also be employed to fabricate the bistable or VAN LC displays, photonic elements e.g., beam steering devices, diffraction gratings, etc.

In spite of having several advantages, further research is required to improve the lens profile, which could include use of certain grayscale masks or control of exposing environments, but these are simple technological issues that can be resolved by further improvement in the technology.

This work is supported by HKUST grants CERG 612310 and RGC 614410.

\section{References}

1. M. Sluijter, A. Herzog, D. K. de Boer, M. P. Krijn, and H. P. Urbach, J. Opt. Soc. Am. B 26, 2035 (2009).

2. M. Ye, B. Wang, M. Kawamura, and S. Sato, Jpn. J. Appl. Phys. 46, 6776 (2007).

3. M. Ye, B. Wang, T. Takahashi, and S. Sato, Opt. Rev. 14, 173 (2007).
4. P. Valley, M. Reza Dodge, J. Schwiegerling, G. Peyman, and N. Peyghambarian, Opt. Lett. 35, 2582 (2010).

5. Y. Lin, M. Chen, and H. Lin, Opt. Express 19, 4714 (2011).

6. P. J. Hands, S. A. Tatarkova, A. K. Kirby, and G. D. Love, Opt. Express 14, 4525 (2006).

7. M. Kawamura, M. Ye, and S. Sato, Jpn. J. Appl. Phys. 44, 6098 (2005).

8. M. Kawamura, M. Ye, and S. Sato, Mol. Cryst. Liq. Cryst. 478, 135 (2007)

9. H. Lin and Y. Lin, Appl. Phys. Lett. 97, 063505 (2010).

10. F. S. Yeung, J. Y. Ho, Y. W. Li, F. C. Xie, O. K. Tsui, P. Sheng, and H. S. Kwok, Appl. Phys. Lett. 88, 51910 (2006).

11. D. W. Berreman and W. R. Heffner, J. Appl. Phys. 52, 3032 (1981).

12. F. Yeung and H. S. Kwok, Appl. Phys. Lett. 88, 063505 (2006).

13. M. C. Tseng, F. Fan, C. Y. Lee, A. Murauski, V. Chigrinov, and H. S. Kwok, J. Appl. Phys. 109, 083109 (2011).

14. J. Ho, V. G. Chigrinov, and H. S. Kwok, Appl. Phys. Lett. 90, 243506 (2007).

15. K. E. Vaughn, M. Sousa, D. Kang, and C. Rosenblatt, Appl. Phys. Lett. 90, 194102 (2007).

16. J. B. Kim, K. C. Kim, H. J. Ahn, B. H. Hwang, J. T. Kim, S. J. Jo, C. S. Kim, H. K. Baik, C. J. Choi, and M. K. Jo, Appl. Phys. Lett. 91, 023507 (2007).

17. T. Uchida, M. Ohgawara, and M. Wada, Jpn. J. Appl. Phys. 19, 2127 (1980).

18. S. T. Wu and D. K. Yang, Fundamentals of Liquid Crystal Devices (Wiley, 2006).

19. H. Ren and S. T. Wu, Opt. Express 14, 11292 (2006). 